\title{
Hole Structures in Nonlocally Coupled Noisy Phase Oscillators
}

\author{
Yoji Kawamura* \\ Department of Physics, Graduate School of Sciences, Kyoto University, Kyoto 606-8502, Japan and \\ The Earth Simulator Center, Japan Agency for Marine-Earth Science and Technology, Yokohama 236-0001, Japan
}

(Dated: December 15, 2006)

\begin{abstract}
We demonstrate that a system of nonlocally coupled noisy phase oscillators can collectively exhibit a hole structure, which manifests itself in the spatial phase distribution of the oscillators. The phase model is described by a nonlinear Fokker-Planck equation, which can be reduced to the complex Ginzburg-Landau equation near the Hopf bifurcation point of the uniform solution. By numerical simulations, we show that the hole structure clearly appears in the space-dependent order parameter, which corresponds to the Nozaki-Bekki hole solution of the complex Ginzburg-Landau equation.
\end{abstract}

PACS numbers: 05.45.Xt, 82.40.Ck

One representative class of coupled oscillator systems is the coupled phase oscillators [1, 2, 3, 4]. For example, globally coupled phase oscillators, such as the Kuramoto model, have attracted the attention of many researchers for a long time [5, 6, 7]. Recently, while coupled phase oscillators on complex networks have been investigated widely [8, 9, 10, 11], nonlocally coupled phase oscillators have also been studied, which exhibit a remarkable class of patterns called chimera where phase-locked oscillators coexist with drifting ones [12, 13, 14, 15, 16].

In this paper, we demonstrate that nonlocally coupled noisy phase oscillators can collectively exhibit a hole structure in their phase distribution. After briefly reviewing several results on the phase model [15, 17], we will present our new findings obtained from numerical simulations of the Langevin-type equation and its corresponding nonlinear Fokker-Planck equation describing the phase model. We will show that a hole structure clearly appears in a properly defined order parameter under suitable conditions, and compare it with the NozakiBekki hole solution of the complex Ginzburg-Landau equation, for which the modulus displays regions of local depression.

A system of nonlocally coupled noisy phase oscillators is described by the following Langevin-type equation (LE) for a phase $\phi(x, t)$ at location $x$ and time $t$ :

$\partial_{t} \phi=\omega+\int_{-\infty}^{\infty} d x^{\prime} G\left(x-x^{\prime}\right) \Gamma\left(\phi(x, t)-\phi\left(x^{\prime}, t\right)\right)+\xi(x, t)$.

Here the first term $\omega$ represents the natural frequency common to all the oscillators, the second term the nonlocal coupling, and the last term the additive noise. The phase coupling function $\Gamma(\phi)$, which is a $2 \pi$-periodic function of $\phi$, satisfies the in-phase condition, i.e., $d \Gamma(\phi) /\left.d \phi\right|_{\phi=0}<0$ [2]. The spatial coupling function $G(x)$ is given by

$$
G(x)=\frac{1}{2} \exp (-|x|),
$$

*Electronic address: kawamura@ton.scphys.kyoto-u.ac.jp which is normalized in the infinite domain. The noise is assumed to be Gaussian-white, whose statistics are specified by

$$
\langle\xi(x, t)\rangle=0, \quad\left\langle\xi(x, t) \xi\left(x^{\prime}, t^{\prime}\right)\right\rangle=2 D \delta\left(x-x^{\prime}\right) \delta\left(t-t^{\prime}\right) .
$$

Equation (1) can be derived from a certain class of reaction-diffusion systems under suitable conditions, using the phase reduction method after adiabatically eliminating a highly diffusive chemical component [15].

In Refs. [15, 17], it was shown that the LE (11) is equivalent to a single-oscillator nonlinear Fokker-Planck equation (FPE) given by

$$
\begin{aligned}
& \frac{\partial f(\psi, x, t)}{\partial t}=-\frac{\partial}{\partial \psi}[V(\psi, x, t) f(\psi, x, t)]+D \frac{\partial^{2} f(\psi, x, t)}{\partial \psi^{2}}, \\
& V(\psi, x, t)=\omega+\int_{-\infty}^{\infty} d x^{\prime} G\left(x-x^{\prime}\right) \\
& \times \int_{0}^{2 \pi} d \psi^{\prime} \Gamma\left(\psi-\psi^{\prime}\right) f\left(\psi^{\prime}, x^{\prime}, t\right),
\end{aligned}
$$

where $f(\psi, x, t)$ is a space-time-dependent single phase distribution function of $\psi$, i.e., the normalized probability density that $\phi(x, t)$ takes a value $\psi$ (see also Refs. [18, 19]).

The phase model (1) is capable of sustaining traveling waves below a critical noise strength, $D=D_{c}$, where the uniform solution of the FPE (4) undergoes a Hopf bifurcation [15, 17]. In the vicinity of this Hopf bifurcation point, we can derive a complex Ginzburg-Landau equation (CGLE)

$$
\partial_{t} A(x, t)=\lambda^{2}\left(D_{c}-D\right) A+d \partial_{x}^{2} A-g|A|^{2} A,
$$

from the FPE (4) by applying the center-manifold reduction method [2], where we introduced the complex amplitude $A(x, t)$ representing the fluctuation of $f(\psi, x, t)$ in the phase direction as

$$
f(\psi, x, t)=\frac{1}{2 \pi}+\frac{1}{2 \pi}\left(A(x, t) e^{i \lambda \psi+i \Omega t}+A^{*}(x, t) e^{-i \lambda \psi-i \Omega t}\right) .
$$


Here $\lambda$ is the wavenumber of the phase fluctuation, $\Omega$ the Hopf frequency, and $A^{*}$ the complex conjugation of $A$. The parameters of the CGLE (6) are given by

$$
\begin{gathered}
D_{c}=\Im \Gamma_{\lambda} / \lambda, \quad \Omega=-\lambda\left(\omega+\Re \Gamma_{\lambda}+\Gamma_{0}\right), \\
d=-i \lambda \Gamma_{\lambda}, \quad g=\frac{\lambda \Gamma_{\lambda}\left(\Gamma_{2 \lambda}+\Gamma_{-\lambda}\right)}{2 \Im \Gamma_{\lambda}-i \Re \Gamma_{\lambda}+i \Gamma_{2 \lambda}},
\end{gathered}
$$

where $\lambda=\arg \max _{l} \Im \Gamma_{l} / l$, and $\Gamma_{l}$ is the Fourier component of the phase coupling function defined by

$$
\Gamma(\psi)=\sum_{l=-\infty}^{\infty} \Gamma_{l} e^{i l \psi}
$$

In what follows, we restrict ourselves to the case of $\lambda=1$ and positive $\Re g$, i.e., the case that the first Fourier component of the phase distribution function has the largest critical noise strength, and the Hopf bifurcation of the uniform solution is supercritical.

Since we assume $\lambda=1$, Eq. (8) and Eq. (9) depend only on the first and the second harmonics of the phase coupling function, $\Gamma_{ \pm 1}$ and $\Gamma_{2}$. In this case, without loss of generality, the phase coupling function can be expressed as

$$
\Gamma(\psi)=-\sin (\psi+\alpha)+u \sin (2 \psi+\beta) .
$$

Now let us introduce a space-time-dependent complex order parameter with modulus $R(x, t)$ and phase $\Theta(x, t)$ as

$$
\begin{aligned}
R(x, t) e^{i \Theta(x, t)} & \equiv \int_{-\infty}^{\infty} d x^{\prime} G\left(x-x^{\prime}\right) e^{i \phi\left(x^{\prime}, t\right)} \\
& =\int_{-\infty}^{\infty} d x^{\prime} G\left(x-x^{\prime}\right) \int_{0}^{2 \pi} d \psi^{\prime} e^{i \psi^{\prime}} f\left(\psi^{\prime}, x^{\prime}, t\right) \\
& =\int_{-\infty}^{\infty} d x^{\prime} G\left(x-x^{\prime}\right) A^{*}\left(x^{\prime}, t\right) e^{-i \Omega t} \\
& \simeq A^{*}(x, t) e^{-i \Omega t}
\end{aligned}
$$

In deriving the last expression, we utilized the fact that the spatial characteristic length of the complex amplitude becomes sufficiently long compared to the nonlocal coupling length near the critical point 15, 17]. Thus, the order parameter corresponds to the complex conjugation of the complex amplitude, which is governed by the CGLE (6). As is well known, CGLE admits the NozakiBekki hole solutions [20, 21, 22, 23, 24, 25], for which the existence of the amplitude degree of freedom is crucial. In the following, we will show that the phase model (1) can exhibit a hole structure in its space-time phase distribution, despite its lack of apparent amplitude variables.

First of all, let us identify the Hopf bifurcation point predicted from the FPE (41) by numerical simulations of the LE (1). In the numerical simulations, our continuous media of size $L$ is replaced with a long array of $N$ oscillators with sufficiently small separation $\Delta x$ between the neighboring oscillators, i.e., $L=N \Delta x$, and the periodic boundary condition is imposed. In the continuous limit, $N \rightarrow \infty$ with $L$ fixed, the Langevin simulation would exactly correspond to the Fokker-Planck simulation, but we can use only finite values of $N$ in actual Langevin simulations. Thus, we must consider the effect of finite-size fluctuations, which comes from the finiteness of the oscillator number within the nonlocal coupling range. Applying the finite-size scaling argument developed in Ref. 26] (see also Ref. [27]) to the space-time-averaged modulus $\langle R\rangle$ of the order parameter, we can obtain a scaling form

$$
N^{1 / 4}\langle R\rangle=F\left(N^{1 / 2}\left(D_{c}-D\right)\right),
$$

where $F$ is a scaling function depending on $N$ and $\left(D_{c}-\right.$ $D)$ only through the combination $N^{1 / 2}\left(D_{c}-D\right)$. We fix our system size $L$ to be 8.0 and vary the number $N$ of the oscillators, i.e., $L=N \Delta x=8.0$. For this Langevin simulation, we fix the parameter values as

$$
\alpha=0.5, \quad u=0.0,
$$

which yield the critical noise strength

$$
D_{c}=\cos (0.5) / 2 \text {, }
$$

and the two essential parameters of the CGLE [2, 23],

$$
c_{1} \equiv \Im d / \Re d=\tan (0.5), \quad c_{2} \equiv \Im g / \Re g=-\tan (0.5) / 2 \text {. }
$$

With these parameter values, spatially uniform oscillations are realized in the Langevin simulation, as expected from the phase diagram of the CGLE 25]. Figure[1 summarizes the numerical results in the rescaled variables, where $N^{1 / 4}\langle R\rangle$ is plotted as a function of $N^{1 / 2}\left(D_{c}-D\right)$ for several values of $N$. All curves collapse onto a single identical curve after rescaling, which agrees well with the prediction based on the FPE (4). This gives good evidence for the existence of the Hopf bifurcation at $D=D_{c}$.

Now we demonstrate that the phase model (1) can exhibit a hole structure. We first carry out a Fokker-Planck simulation. The parameter values are chosen as

$$
\alpha=\arctan (0.5), \quad \beta=2.3, \quad u=1.8,
$$

which give

$$
\begin{gathered}
D_{c}=\cos (\arctan (0.5)) / 2, \\
c_{1} \equiv \Im d / \Re d=0.5, \quad c_{2} \equiv \Im g / \Re g \simeq 2.0 .
\end{gathered}
$$

The corresponding CGLE (6) has stable hole solutions with these parameter values [25]. In our numerical simulations of the FPE (4), periodic boundary conditions are imposed for both phase $\psi$ and space $x$. The intervals of the phase and the space are $2 \pi$ and $L=102.4$, respectively. The pseudo-spectral method with $M=32$ modes is applied for the phase. The number of the spatial grid 


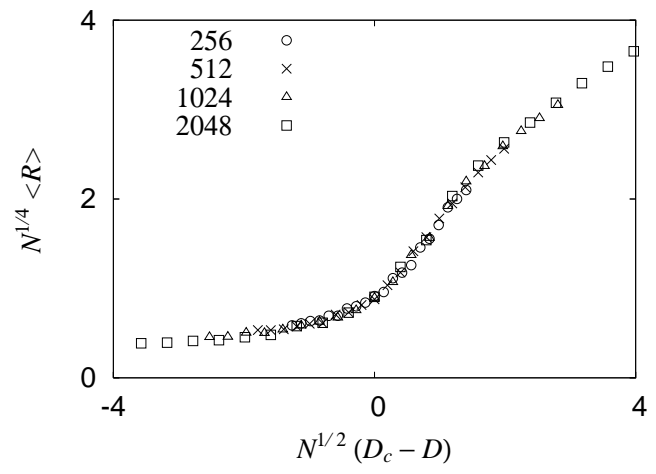

FIG. 1: Dependence of the order-parameter modulus on the noise strength, obtained from the Langevin-type equation (1) using different values of the number of oscillators $N$. The data are plotted using rescaled variables, revealing the scaling function given by Eq. (13).

points is $N=512$, i.e., $\Delta x=0.2$. Our numerical results are unchanged if we further increase the number of modes $M$, the number of grid points $N$, or the system size $L$. The initial condition is given by

$f(\psi, x, t=0)=C_{x}\left[2+B(x) \exp (i \psi)+B^{*}(x) \exp (-i \psi)\right]$,

where $C_{x}$ is a normalization constant, and

$$
B(x)= \begin{cases}4(x-L / 4) / L & (0 \leq x<L / 2) \\ \exp [2 \pi i(x-L / 2) / L] & (L / 2 \leq x<L) .\end{cases}
$$

Note that this $B(x)$ has one "phase singularity" and satisfies the periodic boundary condition. The noise intensity is chosen as $D / D_{c}=0.9$.

Figure 2 displays the spatial profile of the phase distribution function $f(\psi, x, t)$ obtained from a numerical simulation of the FPE (4). Figure 3 displays the spatial profile of the order-parameter modulus (a) and the phase portrait of the order parameter (b), which are obtained from Fig. 2 using the relation given in Eq. (12). We can confirm that a hole structure actually appears in the order parameter. The small bump at the right of the hole is a shock structure due to the collision of counter-propagating plane waves emitted from the hole structure. Once such a hole structure is formed, it stably persists throughout our numerical simulation. This hole structure can be well fitted by a non-propagating Nozaki-Bekki hole solution [23] in the form

$$
W_{\mathrm{H}}(x)=a \tanh [b(x-c)] \exp [i \theta(x)],
$$

$$
d \theta / d x=s \tanh [b(x-c)]
$$

where $a, b, c$, and $s$ are real parameters. These parameters are estimated as $a_{\mathrm{FP}} \simeq 0.45, b_{\mathrm{FP}} \simeq 0.23$, and $s_{\mathrm{FP}} \simeq 0.15$ from the numerical simulation of the FPE (44), while the theoretical values for the reduced CGLE (6) are given by $a_{\mathrm{GL}} \simeq 0.58, b_{\mathrm{GL}} \simeq 0.27$, and $s_{\mathrm{GL}} \simeq 0.12$. The agreement between the simulation and the theory seems reasonable, in consideration of various approximations used in deriving the CGLE (6) from the FPE (4) [29].

We also carried out Langevin simulations with periodic boundary conditions. We prepared an appropriate initial distribution of the oscillators using the hole solution of the FPE (4). Figure 4 displays the snapshot of the local oscillator phase obtained after the initial transient for $L=102.4$ and $N=2^{17}$. The spatial profile of the local oscillator phase well corresponds to the spatial phase distribution shown in Fig. 2. However, this phase distribution corresponding to the hole structure eventually collapses to that corresponding to a plane wave due to the finite-size fluctuation that we mentioned above. The lifetime of the hole structure clearly increases with $N$, so that the hole structure is expected to exist stably in the $N \rightarrow \infty$ limit [30].

In summary, we studied a system of nonlocally coupled noisy phase oscillators based on the LE (11) and its corresponding FPE (4). We confirmed that the onset of the coherence in the order parameter of the LE (1) is identical to the Hopf bifurcation of the FPE (4), using the finitesize scaling relation for the numerical data obtained from the Langevin simulation. We then demonstrated that a stable hole structure can appear in the order parameter calculated from the FPE (4), which corresponds to the non-propagating Nozaki-Bekki hole solution of the CGLE (6). Phase models generally lack the amplitude variables, which are crucial for the hole solutions with phase singularities. In the noisy phase model (1), however, the external random force effectively produces the amplitude degrees of freedom in the phase distribution of the oscillators. The phase model (1) can exhibit yet another interesting phenomenon called noise-induced turbulence 15, 17]. Detailed numerical and theoretical analysis on this phenomenon is recently reported in Ref [28].

\section{Acknowledgments}

The author is grateful to Y. Kuramoto, T. Mizuguchi, H. Nakao, D. Tanaka, and K. Arai for useful discussions.
[1] A. T. Winfree, The Geometry of Biological Time (Springer, New York, 1980; Springer, Second Edition, New York, 2001).

[2] Y. Kuramoto, Chemical Oscillations, Waves, and Tur- bulence (Springer, New York, 1984; Dover, New York, 2003).

[3] A. Pikovsky, M. Rosenblum, and J. Kurths, Synchronization (Cambridge University Press, Cambridge, 2001). 


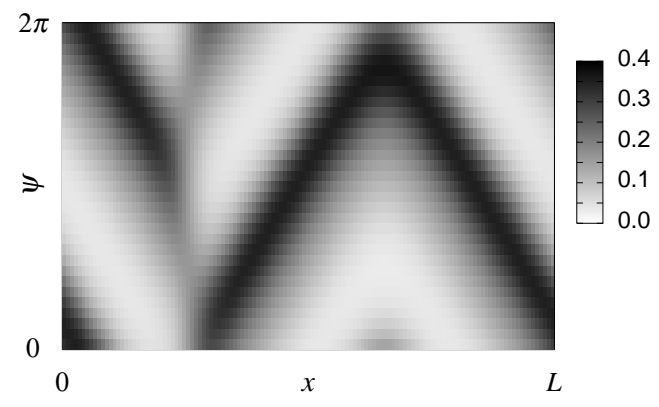

FIG. 2: Instantaneous spatial profile of the phase distribution function $f(\psi, x, t)$ obtained from a numerical simulation of the nonlinear Fokker-Planck equation (4).
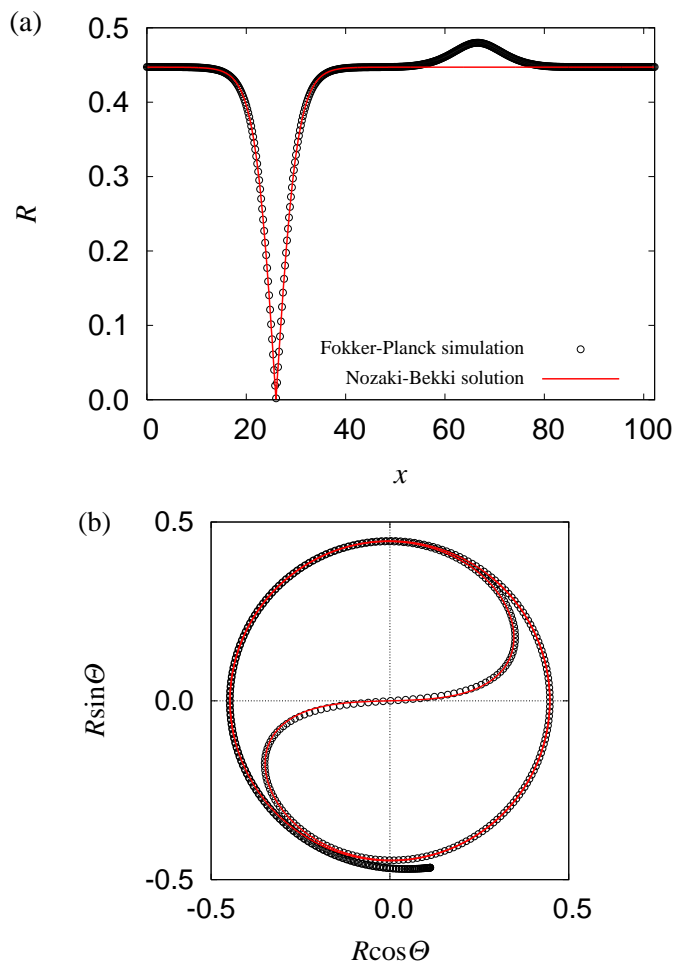

FIG. 3: (Color online) Instantaneous spatial profile of the order-parameter modulus (a). Instantaneous phase portrait of the order parameter (b). They are obtained from Fig. 2 using the relation given in Eq. (12). Solid lines are drawn using the Nozaki-Bekki hole solution of the CGLE given by Eq. (22) and Eq. (23), where the parameter values are estimated as $a_{\mathrm{FP}} \simeq 0.45, b_{\mathrm{FP}} \simeq 0.23, c_{\mathrm{FP}} \simeq 26$, and $s_{\mathrm{FP}} \simeq 0.15$.

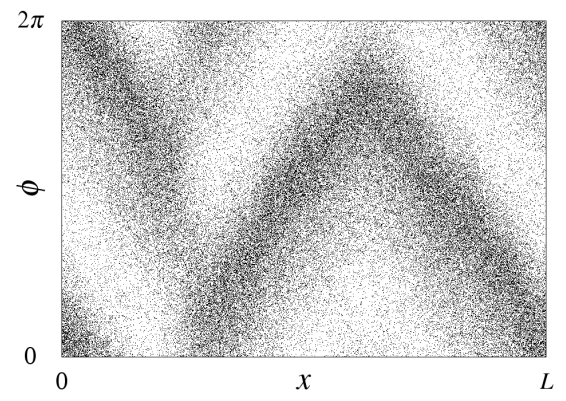

FIG. 4: Instantaneous spatial profile of the local oscillator phase obtained from a numerical simulation of the Langevintype equation (1).
[4] S. C. Manrubia, A. S. Mikhailov, and D. H. Zanette, Emergence of Dynamical Order (World Scientific, Singapore, 2004).

[5] S. H. Strogatz, Physica D 143, 1 (2000).

[6] I. Z. Kiss, Y. M. Zhai, and J. L. Hudson, Science 296, 1676 (2002).

[7] J. A. Acebrón, L. L. Bonilla, C. J. Pérez Vicente, F. Ritort, and R. Spigler, Rev. Mod. Phys. 77, 137 (2005).

[8] H. Kori and A. S. Mikhailov, Phys. Rev. Lett. 93, 254101 (2004); Phys. Rev. E 74, 066115 (2006).

[9] T. Ichinomiya, Phys. Rev. E 70, 026116 (2004).

[10] J. G. Restrepo, E. Ott, and B. R. Hunt, Phys. Rev. E 71, 036151 (2005); Chaos 16, 015107 (2006).

[11] S. Boccaletti, V. Latora, Y. Moreno, M. Chavez, and D.U. Hwang, Phys. Rep. 424, 175 (2006).

[12] Y. Kuramoto and D. Battogtokh, Nonlin. Phenom. Complex Syst. 5, 380 (2002).

[13] D. M. Abrams and S. H. Strogatz, Phys. Rev. Lett. 93, 174102 (2004); Int. J. Bifurcation Chaos 16, 21 (2006).

[14] S. Shima and Y. Kuramoto, Phys. Rev. E 69, 036213 (2004).

[15] Y. Kuramoto, S. Shima, D. Battogtokh, and Y. Shiogai, Prog. Theor. Phys. Suppl. 161, 127 (2006).

[16] Y. Kawamura, Phys. Rev. E 75, 056204 (2007).

[17] Y. Shiogai and Y. Kuramoto, Prog. Theor. Phys. Suppl. 150, 435 (2003).

[18] H. Risken, The Fokker-Planck Equation (Springer, Berlin, 1989).

[19] C. W. Gardiner, Handbook of Stochastic Methods (Springer, Berlin, 1997).

[20] K. Nozaki and N. Bekki, J. Phys. Soc. Jpn. 53, 1581 (1984); N. Bekki and K. Nozaki, Phys. Lett. A 110, 133 (1985).

[21] J. Lega, B. Janiaud, S. Jucquois, and V. Croquette, Phys. Rev. A 45, 5596 (1992).

[22] M. C. Cross and P. C. Hohenberg, Rev. Mod. Phys. 65, 851 (1993).

[23] H. Mori and Y. Kuramoto, Dissipative Structures and Chaos (Springer, Berlin, 1997).

[24] J. Burguete, H. Chaté, F. Daviaud, and N. Mukolobwiez, Phys. Rev. Lett. 82, 3252 (1999).

[25] I. S. Aranson and L. Kramer, Rev. Mod. Phys. 74, 99 (2002).

[26] A. Pikovsky and S. Ruffo, Phys. Rev. E 59, 1633 (1999).

[27] Y. Kawamura and Y. Kuramoto, Phys. Rev. E 69, 016202 (2004); Prog. Theor. Phys. Suppl. 161, 216 (2006).

[28] Y. Kuramoto and Y. Kawamura, J. Korean Phys. Soc. 50, 170 (2007); Y. Kawamura, H. Nakao, and Y. Kuramoto, Phys. Rev. E 75, 036209 (2007).

[29] The main cause of the discrepancy is the CGLE approximation. The center-manifold reduction to the CGLE (6) implicitly assumes that $\Re d$ and $\Re g$ are of the same order [2, 23]. Our parameter condition (17) for a hole solution gives $\Re d / \Re g \simeq 4$, which is rather large.

[30] It is very difficult to precisely determine how the life time of the hole structure increases with the number of oscillators $N$ by numerical simulations of the Langevin-type equation (11). To derive the dependence of the life time of the hole structure on $N$ is a challenging difficult task. For this purpose, investigations on a complex GinzburgLandau equation with external noise would be more suitable. 